\title{
Polarization-Dependent Theory of Two-Wave Mixing in Nonlinear Media, and Application to Dynamical Polarization Control
}

\author{
P. Michel, ${ }^{1}$ E. Kur $\odot,{ }^{2}$ M. Lazarow $\odot,{ }^{2}$ T. Chapman, ${ }^{1}$ L. Divol, ${ }^{1}$ and J. S. Wurtele ${ }^{2}$ \\ ${ }^{1}$ Lawrence Livermore National Laboratory, Livermore, California 94551, USA \\ ${ }^{2}$ Department of Physics, University of California at Berkeley, Berkeley, California 94720-7300, USA
}

(Received 25 January 2020; revised manuscript received 10 March 2020; accepted 1 April 2020; published 19 May 2020)

\begin{abstract}
A scheme for controlling the polarization of a light wave via its interaction with an auxiliary beam in a nonlinear optical medium is proposed. We first present the linear theory of polarization-dependent wave mixing, where the "probe" beam, whose polarization is to be manipulated, is less intense than the auxiliary beam. Then we show that a simple geometrical arrangement, where the auxiliary and probe are crossing at $90^{\circ}$ and the auxiliary is linearly $s$ polarized (orthogonal to the plane of incidence), enables us to control the probe's polarization even when its intensity exceeds the auxiliary's intensity. These schemes are of particular interest when the nonlinear optical medium is a plasma, as it might enable dynamic polarization manipulations at ultrafast timescales and far beyond the optics damage threshold of crystal-based photonics devices.
\end{abstract}

DOI: 10.1103/PhysRevX.10.021039

Subject Areas: Optics, Photonics, Plasma Physics

\section{INTRODUCTION}

Controlling and manipulating the polarization state of light waves is crucial for many applications in various areas of physics and optics research and technology. This can be achieved using passive optical elements, whose internal microscopic structure leads to optical anisotropy and birefringence, or with active elements, where an external field is applied into an optical medium in order to dynamically introduce anisotropy and birefringence. Some well-known examples of active elements include Faraday rotators (polarization rotation via an applied magnetic field in a crystal) or Pockels cells (birefringence induced by an applied electric field in a crystal) [1].

Recently, a new scheme was proposed to manipulate the polarization of a light wave, where a plasma constitutes the optical medium and an auxiliary laser modifies the plasma's optical properties via the nonlinear interaction of the two waves and the plasma. The optical system constituted by the plasma and the auxiliary laser was shown to be anisotropic for a separate "probe" laser beam, thus enabling new concepts of plasma polarizer and plasma wave plate [2]. These two concepts were subsequently demonstrated in proof-of-principle experiments [3,4]. However, in both the theory and experimental demonstrations, polarization mixing was kept in the "linear" regime, i.e., the probe's intensity was kept much smaller than the auxiliary's

Published by the American Physical Society under the terms of the Creative Commons Attribution 4.0 International license. Further distribution of this work must maintain attribution to the author(s) and the published article's title, journal citation, and DOI. intensity, so that the auxiliary beam's polarization state remained unaffected during the interaction. While this made the theory more tractable and the experiments simpler to design, the requirement of an auxiliary beam that is more intense than the beam that is being manipulated is a major hurdle that would prevent the practical applications of these concepts.

In this article, we extend the linear theory of polarizationdependent wave mixing to arbitrary nonlinear media and propose a simple setup that should enable the practical application of these polarization mixing concepts in the "nonlinear" regime, i.e., where the intensity of the probe beam (whose polarization state is being manipulated) can be greater than the intensity of the auxiliary beam (whose role is to control the polarization state of the probe). These schemes should be valid for any optical medium with an intensitydependent refractive index of the form $n=n_{0}+n_{2} I$. We derive linear and nonlinear analytical solutions describing the evolution of the probe's polarization in two dimensions and discuss the concepts of polarizers and Pockels cells based on polarization mixing. These concepts seem particularly attractive for a fast nonlinearity such as ionization in a gas, as this could enable ultrafast polarization manipulation, as well as for interactions in plasmas, as this could enable the realization of plasma-based, and thus essentially indestructible, new photonics devices.

\section{GEOMETRY OF POLARIZATION-DEPENDENT TWO-WAVE MIXING}

In the following, we consider two light waves with wave vectors and frequencies $\left(\boldsymbol{k}_{0}, \omega_{0}\right)$ and $\left(\boldsymbol{k}_{1}, \omega_{1}\right)$, propagating along the directions $\zeta_{0}$ and $\zeta_{1}$ in a nonlinear medium 
characterized by an intensity-dependent index of refraction. The total electric field of the waves is $\tilde{\boldsymbol{E}}(\boldsymbol{r}, t)=\frac{1}{2} \boldsymbol{E}_{0}(\boldsymbol{r}, t) \exp \left[i \psi_{0}\right]+\frac{1}{2} \boldsymbol{E}_{1}(\boldsymbol{r}, t) \exp \left[i \psi_{1}\right]+$ c.c. (the tilde denotes quantities that vary rapidly in time), where $\psi_{0,1}=\boldsymbol{k}_{0,1} \cdot \boldsymbol{r}-\omega_{0,1} t$. The refractive index $n$ of the medium has a nonlinear response to a light wave intensity of the type $n=n_{0}+n_{2} I$, where $n_{0}$ is the linear index and $I=n_{0} c\left\langle\tilde{\boldsymbol{E}}^{2}\right\rangle / 4 \pi$ [1]. Averaging over the fast oscillations from the beat wave gives an index modulation of the form:

$n=n_{0}+\frac{n_{0} n_{2} c}{4 \pi}\left(\frac{1}{2} \boldsymbol{E}_{0} \cdot \boldsymbol{E}_{1}^{*} \exp \left[i\left(\psi_{b}+\phi_{2}\right]+\right.\right.$ c.c. $)$,

where $\psi_{b}=\psi_{0}-\psi_{1}=\boldsymbol{k}_{b} \cdot \boldsymbol{r}-\omega_{b} t$, with $\omega_{b}=\omega_{0}-\omega_{1}$ and $\boldsymbol{k}_{b}=\boldsymbol{k}_{0}-\boldsymbol{k}_{1}$.

The dephasing term $\phi_{2}$ represents the spatial offset (or dephasing) between the beat wave's slowly varying intensity pattern and the resulting refractive index modulation (cf. Fig. 1). Introducing this arbitrary dephasing allows us to describe a wide variety of nonlinear optical processes in various media such as gases, liquids, and crystals. The phase $\phi_{2}$ will generally depend on $\omega_{b}$ and some typical response time of the system, and on the physical origin of the refractive index nonlinearity. In most nonlinear media, for $\omega_{b}=0$ we typically have $\phi_{2}=0(\bmod \pi)$, which prevents energy exchange between the two beams but introduces a phase shift in their electric field (retardation for beam 0 and advance for beam 1 if $\phi_{2}=0$ and vice versa if

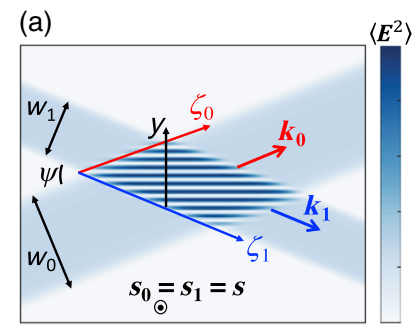

(c) $n_{1 / /}=n_{0}+\delta n_{1}$
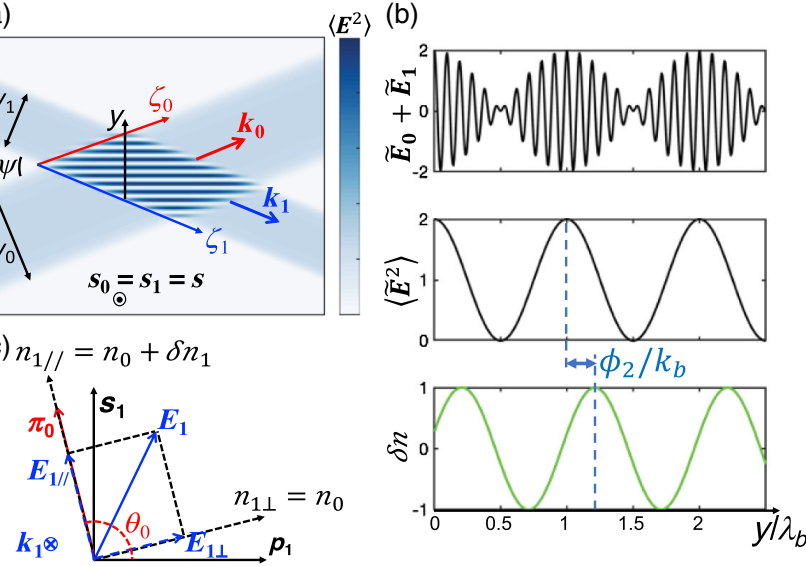

FIG. 1. (a) Interaction geometry between two light waves with wave vectors $\boldsymbol{k}_{0}, \boldsymbol{k}_{1}$ and transverse widths $w_{0}, w_{1}$, showing the beat wave pattern in the interaction volume. (b) The beat wave from $\tilde{\boldsymbol{E}}_{0}+\tilde{\boldsymbol{E}}_{1}$ leads to a time-averaged modulation in intensity $\left\langle\left(\tilde{\boldsymbol{E}}_{0}+\tilde{\boldsymbol{E}}_{1}\right)^{2}\right\rangle$, which in turn generates a refractive index modulation $\delta n$; the refractive index modulation can be spatially shifted from the time-averaged beat wave intensity pattern, by a phase shift $\phi_{2}$. (c) Geometry of the linear wave mixing interaction: when $I_{0} \gg I_{1}$, the probe $\boldsymbol{E}_{1}$ experiences a different refractive index $n_{1 \|}=n_{0}+\delta n_{1}$ along the direction of $\boldsymbol{\pi}_{0}$, the projection of $\boldsymbol{E}_{0}$ in $\left(\boldsymbol{p}_{1}, \boldsymbol{s}_{1}\right)$, whereas the refractive index $n_{1 \perp}$ along the direction orthogonal to $\boldsymbol{\pi}_{0}$ remains unchanged. $\phi_{2}=\pi$, as we see below). On the other hand, when the two waves have different frequencies $\left(\omega_{b} \neq 0\right)$, the low-frequency beat wave has a finite phase velocity $\boldsymbol{v}_{b}=\boldsymbol{k}_{b} \omega_{b} / k_{b}^{2}$ which can often introduce an optical resonance if properly tuned. This is, for example, the case of Brillouin scattering, where the low-frequency wave resonantly drives an acoustic wave in the system $\left(v_{b} \approx c_{s}\right.$, where $c_{s}$ is the sound speed) [1,5]. In this case, we typically have $\phi_{2}=\pi / 2$ $(\bmod \pi)$, and the waves can transfer energy to one another-from beam 0 to beam 1 if $\phi_{2}=\pi / 2$, or vice versa if $\phi_{2}=-\pi / 2$. Specific examples and expressions of $n_{2}$ and $\phi_{2}$ are given in Sec. V.

Next we proceed as in most two-wave mixing analyses, and insert the expressions for the total electric field and the refractive index in the wave equation, $\left[c^{2} \nabla^{2}-n^{2} \partial_{t}^{2}\right] \boldsymbol{E}=0$. Looking for steady-state solutions for the envelopes [i.e., of the form $\boldsymbol{E}_{j}(\boldsymbol{r})$ with $j=0$ or 1], making the usual paraxial and slowly varying envelope approximation (i.e., $\left.\partial_{\zeta_{j}}^{2} E_{j} \ll \nabla_{\perp j}^{2} E_{j} \ll k_{j} \partial_{\zeta_{j}} E_{j}\right)$ and collecting the terms oscillating at $\psi_{0}$ and $\psi_{1}$ gives the following set of coupled equations (cf. e.g., Ref. [1], Sec. 7.4, for more detail on the derivation):

$$
\begin{aligned}
& \frac{\partial \boldsymbol{E}_{0}}{\partial \zeta_{0}}\left(\zeta_{0}, \zeta_{1}\right)=i \frac{k_{0} c}{8 \pi} n_{2} \exp \left[i \phi_{2}\right]\left(\boldsymbol{E}_{0} \cdot \boldsymbol{E}_{1}^{*}\right) \boldsymbol{E}_{1}, \\
& \frac{\partial \boldsymbol{E}_{1}}{\partial \zeta_{1}}\left(\zeta_{0}, \zeta_{1}\right)=i \frac{k_{0} c}{8 \pi} n_{2} \exp \left[-i \phi_{2}\right]\left(\boldsymbol{E}_{1} \cdot \boldsymbol{E}_{0}^{*}\right) \boldsymbol{E}_{0},
\end{aligned}
$$

where we have also assumed that the frequencies of the two light waves are close to each other $\left(\omega_{b} \ll \omega_{0}, \omega_{1}\right)$. However, by keeping the two separate variables $\zeta_{0}$ and $\zeta_{1}$ as well as the full dot products between the electric fields' vectors, we allow both the crossing angle $\psi$ and the polarization states of the two beams to remain arbitrary. The system of equations is nonlinear and in general two dimensional; i.e., the spatial coordinate is decomposed into the nonorthogonal $\left(\zeta_{0}, \zeta_{1}\right)$ basis, where $\left[\zeta_{0}=0, \zeta_{1}=0\right]$ is defined as the beginning of the interaction region between the two beams [cf. Fig. 1(a)].

In order to account for polarization effects, the transverse fields are decomposed into their $p$ and $s$ components using the Jones vector notation, i.e.,

$$
\left|\mathcal{E}_{j}\right\rangle=\left(\begin{array}{c}
E_{j p} \\
E_{j s}
\end{array}\right)
$$

(where $j=0$ or 1 ). Here $\boldsymbol{s}=\boldsymbol{k}_{0} \times \boldsymbol{k}_{1} /\left|\boldsymbol{k}_{0} \times \boldsymbol{k}_{1}\right|$ is the unit vector perpendicular to the plane of incidence of the two light waves (cf. Fig. 1), and $\boldsymbol{p}_{j}=\boldsymbol{k}_{j} \times \boldsymbol{s} / \boldsymbol{k}_{j}$ is the unit vector in the polarization plane of beam $j$ that is in the plane of incidence. The "bra" denotes the conjugate transpose, i.e., $\left\langle\mathcal{E}_{j}\right|=\left(E_{j p}^{*} E_{j s}^{*}\right)$, so that $\left\langle\mathcal{E}_{j} \mid \mathcal{E}_{j}\right\rangle=\left|E_{j}\right|^{2}$. The interaction volume between the two beams (characterized by the 
beat wave fringes in Fig. 1) is supposed to be fully contained inside the nonlinear medium. We consider the two-dimensional case only: since the interaction takes place in the plane of incidence, results in 3D can simply be obtained by repeating the $2 \mathrm{D}$ solutions along the $s$ direction.

Similar to Ref. [2], we multiply Eqs. (2) and (3) by $\left(\boldsymbol{p}_{0}, \boldsymbol{s}\right)$ and $\left(\boldsymbol{p}_{1}, \boldsymbol{s}\right)$, respectively, to obtain the following coupled equations in Jones's vector form describing the nonlinear interaction and polarization mixing between the two light waves:

$$
\begin{aligned}
& \partial_{\zeta_{0}}\left|\mathcal{E}_{0}\right\rangle=\gamma P\left|\mathcal{E}_{1}\right\rangle\left\langle\mathcal{E}_{1}|P| \mathcal{E}_{0}\right\rangle, \\
& \partial_{\zeta_{1}}\left|\mathcal{E}_{1}\right\rangle=-\gamma^{*} P\left|\mathcal{E}_{0}\right\rangle\left\langle\mathcal{E}_{0}|P| \mathcal{E}_{1}\right\rangle,
\end{aligned}
$$

where

$$
P=\left(\begin{array}{cc}
\cos (\psi) & 0 \\
0 & 1
\end{array}\right)
$$

is the projection matrix of one beam's electric field onto the other's plane of polarization [ $\psi$ is the crossing angle between the beams, cf. Fig. 1(a)], and $\gamma=$ $i k_{0} c n_{2} \exp \left[i \phi_{2}\right] / 8 \pi$.

\section{LINEAR REGIME OF POLARIZATION MIXING}

In the linear regime, i.e., when one of the two waves (say, $E_{0}$ ) is much more intense than the other and unaffected by the interaction, the situation reduces to a $1 \mathrm{D}$ problem, as the "probe" beam $\left|\mathcal{E}_{1}\right\rangle$ will be modified in amplitude and/or polarization state until it leaves the interaction volume after propagating through a distance $w_{0} / \sin (\psi)$ [cf. Fig. 1(a)]. Equation (5) can then be solved analytically, as shown in Ref. [2]: the solution for a propagation from 0 to $L$ can be expressed as $\left|\mathcal{E}_{1}(L)\right\rangle=\exp \left[M_{0}\right]\left|\mathcal{E}_{1}(0)\right\rangle$, where $M_{0}=$ $-\gamma^{*} P\left|\mathcal{E}_{0}\right\rangle\left\langle\mathcal{E}_{0}\right| P$ is a $2 \times 2$ matrix. The matrix exponential can easily be calculated using standard techniques, giving the following result:

$$
\left|\mathcal{E}_{1}(L)\right\rangle=R\left(-\theta_{0}\right)\left(\begin{array}{cc}
\exp \left[-\gamma^{*}\left|\pi_{0}\right|^{2} L\right] & 0 \\
0 & 1
\end{array}\right) R\left(\theta_{0}\right)\left|\mathcal{E}_{1}(0)\right\rangle,
$$

where

$$
\left|\pi_{0}\right\rangle=P\left|\mathcal{E}_{0}\right\rangle=\left(\begin{array}{c}
E_{0 p} \cos (\psi) \\
E_{0 s}
\end{array}\right)
$$

is the projection of $\boldsymbol{E}_{0}$ onto the plane $\left(\boldsymbol{p}_{1}, \boldsymbol{s}_{1}\right)$ such that $\left|\pi_{0}\right|^{2}=\left\langle\pi_{0} \mid \pi_{0}\right\rangle=\cos ^{2}(\psi)\left|E_{0 p}\right|^{2}+\left|E_{0 s}\right|^{2}$, and $R\left(\theta_{0}\right)$ is the rotation matrix by $\theta_{0}$, the angle between $\boldsymbol{p}_{1}$ and $\boldsymbol{\pi}_{0}$ [cf. Fig. 1(c)].
When $\boldsymbol{E}_{0}$ is linearly polarized, this result simply means that the component of $\boldsymbol{E}_{1}$ aligned with $\boldsymbol{\pi}_{0}$ is multiplied by $\exp \left[-\gamma^{*}\left|\pi_{0}\right|^{2} L\right]$, whereas the component perpendicular to $\pi_{0}$ stays unaffected. This is equivalent to having a refractive index $n_{1 \|}$ for the component of $\boldsymbol{E}_{1}$ aligned with $\boldsymbol{\pi}_{0}$, and a different index $n_{1 \perp}$ for the component perpendicular to $\boldsymbol{\pi}_{0}$, with

$$
\begin{aligned}
& n_{1 \|}=n_{0}+\exp \left[-i \phi_{2}\right] n_{2} I_{0 \pi}, \\
& n_{1 \perp}=n_{0},
\end{aligned}
$$

where $I_{0 \pi}=\cos ^{2}(\psi) I_{0 p}+I_{0 s}$ and $I_{0 p, s}=n_{0} c\left|E_{0 p, s}\right|^{2} / 8 \pi$ is the auxiliary beam's intensity along $p, s$ [cf. Fig. 1(c)]. Depending on whether $\phi_{2}=\pi / 2(\bmod \pi)$ (i.e., the refractive index perturbation $\delta n_{1}=\exp \left[-i \phi_{2}\right] n_{2} I_{0 \pi}$ is purely imaginary) or $0(\bmod \pi)\left(\delta n_{1}\right.$ is real $)$, the probe's electric field component $\boldsymbol{E}_{1 \|}$ aligned with $\boldsymbol{\pi}_{0}$ will be modified in amplitude (due to energy exchange with the auxiliary) or in phase. This was the basis for the proposal [2] and subsequent demonstration [3,4] of new concepts of polarizers and wave plates based on wave mixing in plasmas.

These concepts are applicable to other nonlinear media as well. For $\phi_{2}=0$, the presence of the auxiliary beam transforms the nonlinear system into a tunable wave plate (i.e., a Pockels cell), with its slow axis along $\boldsymbol{\pi}_{0}$ and a dephasing that can be dynamically tuned by adjusting $I_{0}$. For example, a half-wave plate is obtained for a dephasing of $\pi$, which occurs when the propagation distance reaches $L_{\pi}=\lambda_{0} /\left(2 n_{2} I_{0 \pi}\right)$.

On the other hand, when $\phi_{2}=-\pi / 2$, we have $\delta n_{1}=-\mathrm{in}_{2} I_{0 \pi}$, i.e., the probe's component aligned with $\boldsymbol{\pi}_{0}$ undergoes an exponential decay as it propagates, due to a transfer of energy to the auxiliary. The component perpendicular to $\pi_{0}$ remains unaffected, so the system effectively acts as a polarizer along the direction perpendicular to $\pi_{0}$, with a power extinction of $\exp [-2 \pi] \approx$ $0.2 \%$ after each propagation through a distance $L_{\pi}=$ $\lambda_{0} /\left(2 n_{2} I_{0 \pi}\right)$ (since the field component decays by $\exp [-\pi]$ ).

To summarize, if the probe propagates through an interaction distance $L$, then

(i) in a medium with $\phi_{2}=0$, the system acts as a Pockels cell with a dephasing $\Delta \varphi=2 \pi n_{2} I_{0 \pi} L / \lambda_{0}$ between the slow axis (aligned with $\boldsymbol{\pi}_{0}$ ) and the fast axis (orthogonal to $\boldsymbol{\pi}_{0}$ );

(ii) in a nonlinear medium with $\phi_{2}=-\pi / 2$, the auxiliary transforms the medium into a polarizer along the direction orthogonal to $\boldsymbol{\pi}_{0}$ with a power extinction ratio $\varepsilon=\exp \left[-4 \pi n_{2} I_{0 \pi} L / \lambda_{0}\right]$.

\section{NONLINEAR REGIME AND POLARIZATION CONTROL FOR $I_{1} \geq I_{0}$}

If the probe's intensity becomes large enough to affect the auxiliary's polarization, the polarization mixing 


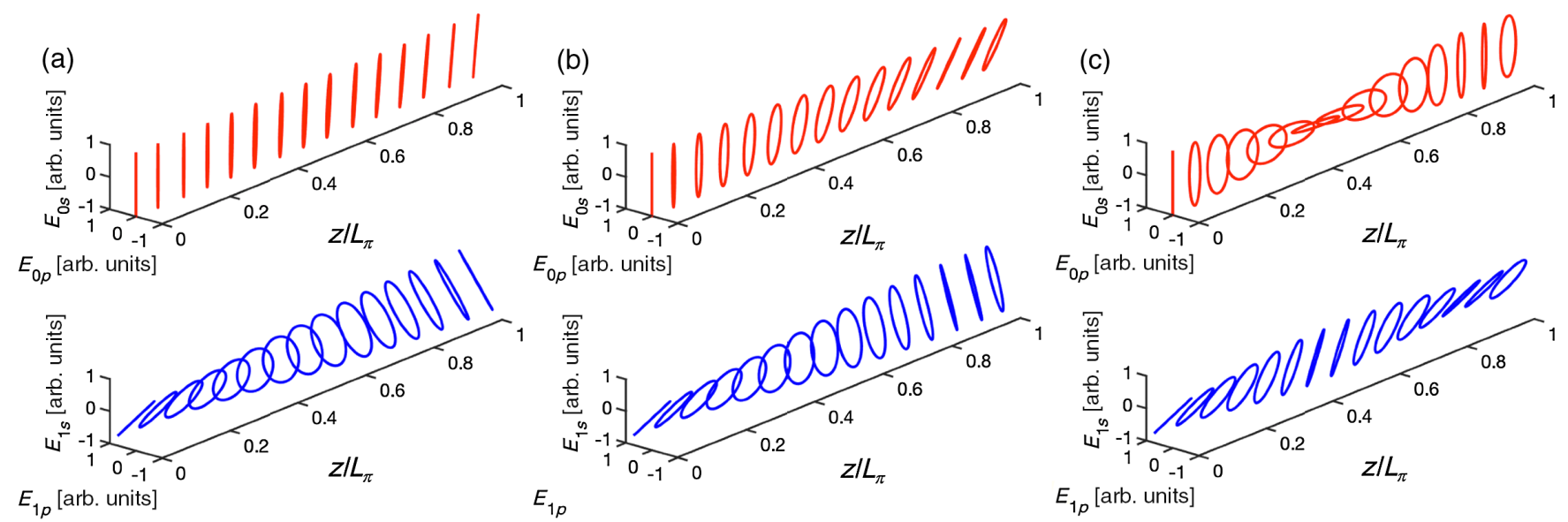

FIG. 2. Transition from linear to nonlinear polarization mixing in $1 \mathrm{D}$ for the simple case of a "wave plate" with the auxiliary $\boldsymbol{E}_{0}$ initially $s$ polarized and the probe linearly polarized at $45^{\circ}$, for $\psi=10^{\circ}$ and (a) $I_{1}=0.1 I_{0}$, (b) $I_{1}=0.5 I_{0}$, (c) $I_{1}=2 I_{0}$. The upper (lower) plots represent the auxiliary's (probe's) polarization state versus $z$.

becomes nonlinear. This is illustrated in Fig. 2, which shows the interaction of an $s$-polarized auxiliary beam with a probe linearly polarized at $45^{\circ}$ for $\phi_{2}=0$ (i.e., a "Pockels cell"). This was calculated by numerically solving the nonlinear system of Eqs. (4) and (5) for a small crossing angle $\left(\psi=10^{\circ}\right)$ at three different ratios of intensity between the two beams: (a) with $I_{1}=0.1 I_{0}$, (b) $I_{1}=0.5 I_{0}$, and (c) $I_{1}=2 I_{0}$. The coordinate $z$ is taken as the bisector between $\zeta_{0}$ and $\zeta_{1}$.

In the first case [Fig. 2(a)], the probe reaches a dephasing of $\pi$ at $z=L_{\pi}$ (half-wave plate), as expected from the linear theory, while the auxiliary remains largely unaffected by the interaction. However, as the probe intensity becomes close to the auxiliary's intensity in Fig. 2(b), the auxiliary's polarization state begins to develop some ellipticity and the probe does not reach a full $90^{\circ}$ rotation of its polarization at $z=L_{\pi}$. This is because the auxiliary's component aligned with the probe (i.e., at $-45^{\circ}$ in the plane $\left[p_{1}, s_{1}\right]$ ) experiences a phase delay compared to the component perpendicular to the probe (at $+45^{\circ}$ in $\left[p_{1}, s_{1}\right]$ ). Finally, when the intensity of the probe actually exceeds the auxiliary's intensity [Fig. 2(c)], the higher probe intensity leads to a phase retardation of the auxiliary by $\sim \pi$ (i.e., a $\pi / 2$ polarization rotation) by $z \simeq 0.4 L_{\pi}$. On the other hand, the probe has not experienced much dephasing because the auxiliary has changed its polarization over too short of a distance. In other words, the probe and auxiliary have essentially swapped their roles.

While this seems to indicate that a beam's polarization can only be controlled via a more intense auxiliary beam, there is in fact a simple geometrical arrangement that allows us to control a probe's polarization state by using a less intense auxiliary beam. As we now demonstrate, the solution consists in crossing the two beams at $90^{\circ}$ and using an $s$-polarized auxiliary beam, and must be treated in the nonlinear regime of polarization mixing.
For clarity we will denote $x$ and $y$ the propagation directions of the beams 1 and 0 , respectively. Beam 1 is again the probe, i.e., the beam whose polarization state we wish to manipulate, and beam 0 is the auxiliary (cf. Fig. 3). The fields are expressed as $\tilde{\boldsymbol{E}}_{0}(x, y)=\frac{1}{2} \boldsymbol{E}_{0}(x, y) \exp \left[i\left(k_{0} y-\omega_{0} t\right)\right]+$ c.c. and $\tilde{\boldsymbol{E}}_{1}(x, y)=$ $\frac{1}{2} \boldsymbol{E}_{1}(x, y) \exp \left[i\left(k_{1} x-\omega_{1} t\right)\right]+$ c.c. Since $\boldsymbol{E}_{0}$ is polarized in $(x, z)$ and $\boldsymbol{E}_{1}$ is polarized in $(y, z)$, the Jones vectors are

$$
\left|\mathcal{E}_{0}(x, y)\right\rangle=\left(\begin{array}{c}
E_{0 x} \\
E_{0 z}
\end{array}\right)
$$

and

$$
\left|\mathcal{E}_{1}(x, y)\right\rangle=\left(\begin{array}{c}
E_{1 y} \\
E_{1 z}
\end{array}\right)
$$

(a)

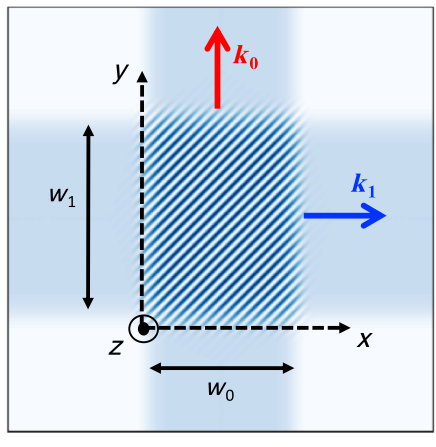

$\left\langle\widetilde{E}^{2}\right\rangle \quad$ (b)

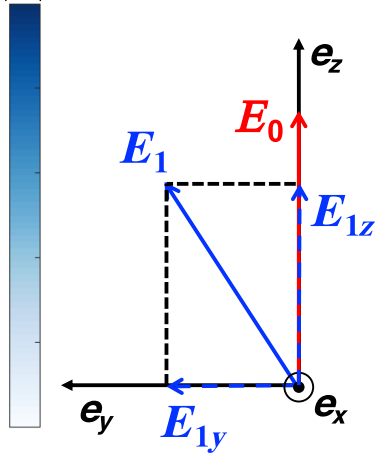

FIG. 3. (a) Geometry for $90^{\circ}$ interaction between the two beams. (b) For an $s$-polarized auxiliary beam (i.e., $\boldsymbol{E}_{0}$ parallel to $z$ ), the $E_{1 z}$ component can be modified in amplitude and/or in phase by the coupling with $E_{0}$, whereas $E_{1 y}$ remains unaffected. 
With the projection matrix $P$ now equal to

$$
\left(\begin{array}{ll}
0 & 0 \\
0 & 1
\end{array}\right)
$$

the coupled equations (4) and (5) simplify to

$$
\begin{aligned}
\partial_{y}\left|\mathcal{E}_{0}\right\rangle & =\left(\begin{array}{c}
0 \\
\gamma\left|E_{1 z}\right|^{2} E_{0 z}
\end{array}\right), \\
\partial_{x}\left|\mathcal{E}_{1}\right\rangle & =\left(\begin{array}{c}
0 \\
-\gamma^{*}\left|E_{0 z}\right|^{2} E_{1 z}
\end{array}\right) .
\end{aligned}
$$

This shows that the $\psi=\pi / 2$ geometry prevents the $p$ components of the two waves, which are now orthogonal to each other, from interacting. Therefore, if the auxiliary beam is initially $s$ polarized, then its $p$ component will remain 0 throughout the interaction (within the limit of our infinite $F / \#$ approximation), and therefore its polarization state will remain $s$.

Equations (9) and (10) are coupled via their two $s$ (i.e., $z$ ) components only. The system of coupled equations is

$$
\begin{aligned}
& \partial_{y} E_{0 z}(x, y)=\gamma\left|E_{1 z}\right|^{2} E_{0 z}, \\
& \partial_{x} E_{1 z}(x, y)=-\gamma^{*}\left|E_{0 z}\right|^{2} E_{1 z} .
\end{aligned}
$$

A similar system of equations was solved previously, in Refs. [6,7]. Following a similar procedure, we obtain the following solutions for the intensities:

$$
\begin{aligned}
& I_{0 z}(\hat{x}, \hat{y})=\frac{I_{0 i}}{1+e^{\hat{x}}\left(e^{\hat{y}}-1\right)}, \\
& I_{1 z}(\hat{x}, \hat{y})=\frac{I_{1 i}}{1+e^{-\hat{y}}\left(e^{-\hat{x}}-1\right)},
\end{aligned}
$$

where $I_{0 i}=I_{0 z}(\hat{x}, 0)$ and $I_{1 i}=I_{1 z}(0, \hat{y})$ are the $z$-component intensities at the boundaries for the two beams (as they enter the interaction region, cf. Fig. 3), and the normalized spatial coordinates are defined as $\hat{x}=4 \pi n_{2} I_{0 i} \sin \left(\phi_{2}\right) x / \lambda_{0}$ and $\hat{y}=4 \pi n_{2} I_{1 i} \sin \left(\phi_{2}\right) y / \lambda_{0}$. Likewise, the following expressions for the phases $\varphi_{0}, \varphi_{1}$ [such that $\left.E_{0,1 z}(x, y)=\left|E_{0,1 z}(x, y)\right| \exp \left[i \varphi_{0,1}(x, y)\right]\right]$ can be obtained:

$$
\begin{aligned}
\varphi_{0}(\hat{x}, \hat{y}) & =\frac{1}{2} \cot \left(\phi_{2}\right) \ln \left[1+e^{\hat{x}}\left(e^{\hat{y}}-1\right)\right], \\
\varphi_{1}(\hat{x}, \hat{y}) & =-\frac{1}{2} \cot \left(\phi_{2}\right) \ln \left[1+e^{-\hat{y}}\left(e^{-\hat{x}}-1\right)\right] .
\end{aligned}
$$

Combining the expressions for the amplitudes and phases, we finally get the following expressions for the Jones vectors at the exit boundaries of each beam (at $x=w_{0}$ for beam 1 and $y=w_{1}$ for beam 0 ):

$$
\begin{aligned}
& \left|\mathcal{E}_{0}\left(x, w_{1}\right)\right\rangle=\left(\begin{array}{cc}
1 & 0 \\
0 & e^{\left(1-i \cot \phi_{2}\right)} f_{0}\left(\hat{x}, w_{1}\right)
\end{array}\right)\left|\mathcal{E}_{0}(x, 0)\right\rangle, \\
& \left|\mathcal{E}_{1}\left(w_{0}, y\right)\right\rangle=\left(\begin{array}{cc}
1 & 0 \\
0 & e^{\left(1+i \cot \phi_{2}\right)} f_{1}\left(w_{0}, \hat{y}\right)
\end{array}\right)\left|\mathcal{E}_{1}(0, y)\right\rangle,
\end{aligned}
$$

with

$$
\begin{aligned}
& f_{0}(\hat{x}, \hat{y})=\left[1+e^{\hat{x}}\left(e^{\hat{y}}-1\right)\right]^{-1 / 2}, \\
& f_{1}(\hat{x}, \hat{y})=\left[1+e^{-\hat{y}}\left(e^{-\hat{x}}-1\right)\right]^{-1 / 2} .
\end{aligned}
$$

Note that since the interaction is limited to the domain $(x \geq 0, y \geq 0)$ per our definition of the coordinate system, and assuming the interaction volume is finite, these expressions will not lead to singularities.

Like in the linear regime, the most interesting cases for polarization manipulation are when $\phi_{2}=0$ or $\pi / 2$ $(\bmod \pi)$, corresponding to dephasing without energy exchange and vice versa. For any other value of $\phi_{2}$, both the amplitude and phase of the electric fields are modified.

When $\phi_{2}=0$, we get $I_{0 z}(x, y)=I_{0 i}$ and $I_{1 z}(x, y)=I_{1 i}$ i.e., the intensities remain constant throughout the interaction region (no energy exchange), but the fields will experience a dephasing (modification of the real part of the refractive index). The expressions for the phases can be obtained either by rederiving from the coupled equations (11) and (12) or by Taylor expanding the previous solutions in Eqs. (15) and (16) for $\phi_{2} \rightarrow 0$, giving

$$
\begin{gathered}
\varphi_{0}(x, y)_{\phi_{2}=0}=2 \pi n_{2} I_{1 i} y / \lambda_{0}, \\
\varphi_{1}(x, y)_{\phi_{2}=0}=2 \pi n_{2} I_{0 i} x / \lambda_{0} .
\end{gathered}
$$

This shows an important property of the system, which is that the phase of each beam remains constant across its transverse direction when $\phi_{2}=0$, and depends only on the propagation distance ( $x$ for beam 1 and $y$ for beam 0 ). In other words, each beam experiences a different refractive index along its $z$ component while propagating through the interaction volume:

$$
\begin{aligned}
& n_{0 z}=n_{0}+n_{2} I_{1 i}, \\
& n_{1 z}=n_{0}+n_{2} I_{0 i} .
\end{aligned}
$$

Since the intensity of the auxiliary beam is unaffected by the probe for $\phi_{2}=0$, the change in refractive index caused by the auxiliary remains uniform. On the other hand, the index experienced along the $p$ component of each beam remains equal to $n_{0}$. Therefore, for an auxiliary beam (beam 0 with our notations) initially $s$ polarized, the probe beam will experience the exact same refractive index change as in the linear case [Eqs. (7) and (8)], with a uniform dephasing across its transverse direction-meaning that 
the interaction will not lead to spatial variations in the polarization of the probe across its spot, at least if the spot profile is appropriately shaped along the $z$ direction as well (which we discuss in the next section). Just like in the linear case, the auxiliary beam turns the nonlinear medium into a half-wave plate for a propagation distance (i.e., an auxiliary beam's spot size $\left.w_{0}\right)$ equal to $L_{\pi}(\psi=\pi / 2)=\lambda_{0} /\left(2 n_{2} I_{0 s}\right)$. And more important, since the auxiliary remains $s$ polarized, the result still holds regardless of the probe intensity, i.e., even for $I_{1} \geq I_{0}$. The auxiliary beam does experience a change in refractive index as well, which would be even larger than the one experienced by the probe when $I_{1} \geq I_{0}$; however, since its polarization state remains $s$, this will not affect the birefringence experienced by the probe, which depends only on the auxiliary beam's intensity (not its phase).

Next, for $\phi_{2}=-\pi / 2$ and an $s$-polarized auxiliary beam, the probe will experience a decay of its $s$ (i.e., $z$ ) component as it propagates through the interaction volume, whereas its $p$ (i.e., $y$ ) component is unaffected. The system thus acts again as a polarizer along the probe's $p$ direction, similar to the linear case-except that now the $90^{\circ}$ crossing geometry and $s$-polarized auxiliary extend the polarizer concept to the nonlinear regime where $I_{1} \geq I_{0}$. The two concepts of Pockels cell and polarizer are illustrated in Fig. 4.

One difference from the linear case is that, unlike for the $\phi_{2}=0$ (wave plate) situation, the polarizer extinction ratio $\varepsilon$ does depend on the transverse direction $y$ : indeed, at the exit plane $x=w_{0}$ the extinction ratio, defined as $\varepsilon=I_{1 z}\left(w_{0}, y\right) / I_{1 i}$, is given by (with $\phi_{2}=-\pi / 2$ )

$$
\varepsilon(y)=\frac{\exp \left[-y / L_{1}\right]}{\exp \left[-y / L_{1}\right]-1+\exp \left[w_{0} / L_{0}\right]},
$$

where $L_{0}=\lambda_{0} /\left(4 \pi n_{2} I_{0 i}\right)$ and $L_{1}=\lambda_{0} /\left(4 \pi n_{2} I_{1 i}\right)$.

However, the $y$ dependence is not necessarily a major problem, since that expression shows that $\varepsilon$ will become arbitrarily close to 0 for any $y$ when $I_{0 i} \rightarrow \infty$-in other words, the extinction ratio can in theory be made arbitrarily small as long as the "auxiliary strength," $\propto n_{2} I_{0 i} w_{0} / \lambda_{0}$, is large enough.
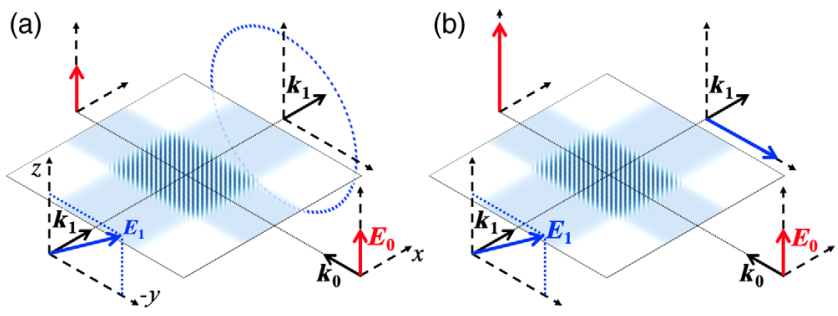

FIG. 4. Illustration of the concepts of polarization control using nonlinear wave mixing at $90^{\circ}$ (a) for $\phi_{2}=0$ and $4 n_{2} I_{0} w_{0} / \lambda_{0}=1$, quarter-wave plate, and (b) for $\psi_{2}=-\pi / 2$, polarizer [the extinction ratio depends on $w_{0}$ and is nonuniform across the transverse direction $y-\mathrm{cf}$. Eq. (25) and Fig. 5].
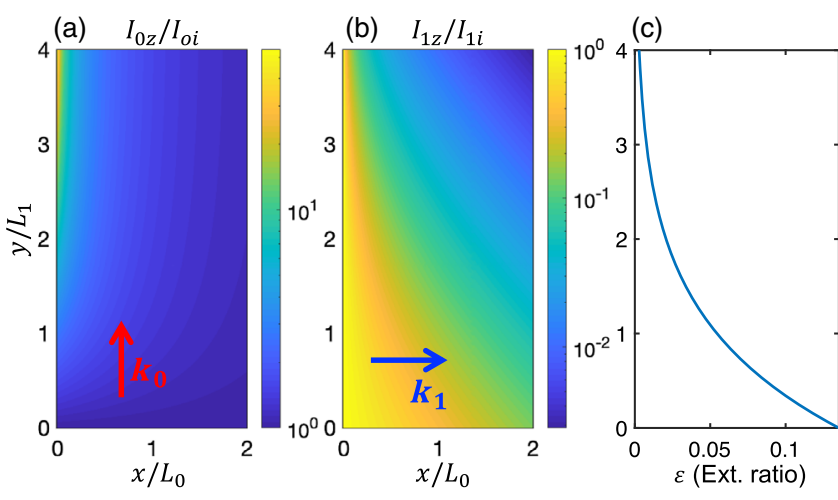

FIG. 5. Spatial nonuniformity of the nonlinear polarizer in the $90^{\circ}$ crossing geometry. (a) Intensity of the auxiliary beam (propagating towards $y>0$ ), (b) intensity of the probe (propagating toward $x>0$ ), (c) extinction ratio $\varepsilon(y)=I_{1 z}\left(w_{0}, y\right) / I_{1 i}$ [i.e., lineout of (b) at $x / L_{0}=2$ ].

This nonlinear 2D dependence is illustrated in Fig. 5, where we have represented the $2 \mathrm{D}$ evolution of the $s$ components of the intensities with the same geometry as in Fig. 3, with an interaction rectangle (assuming flattop beam profiles) of dimensions $w_{0}=2 L_{0}$ and $w_{1}=4 L_{1}$, and a ratio of initial intensities between the two beams set to 1 for figure clarity (this would, for example, represent the case where the probe has twice more power than the auxiliary, assuming the beams have the same size along $z$ ). This illustrates the 2D geometry of the energy transfer nonlinearity: the auxiliary gains energy as it propagates along $y>0$ and primarily on its left side, which is where the probe is coming from. On the other hand, the probe gets depleted as it propagates toward $x>0$, and primarily near the top since this is where the auxiliary has accumulated more spatial growth, making the coupling (and probe depletion) stronger.

\section{EXAMPLES AND DISCUSSION}

In most nonlinear media (e.g., with a Kerr effect), the refractive index modulation is aligned with the beat wave pattern $\left(\phi_{2}=0\right)$. However, when a frequency shift $\omega_{b}$ is present between the two beams, a finite spatial shift can appear if $\omega_{b}$ becomes comparable to $1 / \tau$, where $\tau$ is a typical response time of the medium (e.g., in the sense of a Debye relaxation, cf. [1]). In this case, $n_{2}$ in our Eq. (1) should be replaced by $n_{2} / \sqrt{1+\left(\omega_{b} \tau\right)^{2}}$, and the dephasing is simply given by $\phi_{2}=\arctan \left(\omega_{b} \tau\right)$. This recovers the expression from Ref. [1], and basically shows that when the beat frequency $\omega_{b}$ becomes comparable to $1 / \tau$, the dephasing $\phi_{2}$ is not zero anymore, which leads to energy exchange between the beams.

On the other hand, when the beams' frequency difference $\omega_{b}$ results in a phase velocity of the interference fringes $\boldsymbol{v}_{b}=\boldsymbol{k}_{b} \omega_{b} / k_{b}^{2}$, which matches a characteristic velocity of the nonlinear optical system, the dephasing 
becomes $\phi_{2}=\pi / 2(\bmod \pi)$; i.e., the beams can transfer energy to one another and there is no dephasing of their electric fields. The direction of the energy transfer depends on the sign of the frequency shift, such that the highfrequency beam (in the frame of the medium, in case it is moving) will be giving its energy to the other (lowerfrequency) beam. This situation includes stimulated Brillouin scattering (SBS) in various media, such as liquids, gases, or plasmas. It can easily be verified that our expression for the refractive index modulation in Eq. (1) matches the standard SBS results by simply setting $\phi_{2}=$ $-\pi / 2$ and $n_{2}=\frac{1}{2} g c / \omega_{0}$, where $g$ is the SBS gain factor (in $\mathrm{m} / \mathrm{W})$ such that $I_{1}(z+L)=I_{1}(z) \exp \left[-g I_{0} L\right]$.

The effect can also be achieved by using two beams of identical frequencies in a moving medium (e.g., via a flow), relying on the Doppler shift $\Delta \omega=-\boldsymbol{k}_{b} \cdot \boldsymbol{V}$, where $\boldsymbol{V}$ is the velocity of the medium in the laboratory frame. Note that our analysis also encompasses stimulated Raman scattering (SRS). Since SRS usually involves larger frequency shifts than for SBS, one would have to keep track of the frequency ratio between the two beams in the analytical derivations. Otherwise the main results and conclusions remain the same.

Plasmas or partially ionized gases are particularly attractive media for these concepts since the response time can be extremely fast (e.g., of the order of the ionization time if working with gases near the ionization threshold, which can be subfemtosecond [8-10]), allowing ultrafast polarization manipulation, and because these media could allow manipulation of light at extreme fluences without sustaining optics damage like crystal-based systems [11].

In plasmas, the nonlinear index of refraction and the dephasing take the following form:

$$
\begin{aligned}
& n_{2}=\frac{\left|F_{\chi}\right| k_{b}^{2}}{4 k_{0}^{2} n_{c} m_{e} c^{3}}, \\
& \phi_{2}=\arg \left(F_{\chi}\right),
\end{aligned}
$$

where $n_{c}$ is the critical density for the laser wavelength, and $F_{\chi}=\chi_{e}\left(1+\chi_{i}\right) /\left(1+\chi_{e}+\chi_{i}\right)$, where $\chi_{e}\left(\omega_{b}, \boldsymbol{k}_{b}\right)$ and $\chi_{i}\left(\omega_{b}, \boldsymbol{k}_{b}\right)$ are the electron and ion susceptibilities. These expressions recover our previous results from Ref. [2]. In the fluid approximation and when the frequency shift is small, $\omega_{b} \ll \omega_{0}, \omega_{1}$, the susceptibilities can be approximated by $\chi_{e} \approx 1 /\left(k_{b} \lambda_{D}\right)^{2}$, where $\lambda_{D}$ is the Debye length, and $\chi_{i} \approx-\omega_{\mathrm{pi}}^{2} / \omega^{2}(1+2 i \nu / \omega)$, where $\omega_{\mathrm{pi}}$ is the plasma ion frequency and $\nu$ is the ion acoustic wave damping. We finally get

$$
\begin{aligned}
& \operatorname{Re}\left[F_{\chi}\right] \approx \frac{\omega_{\mathrm{pi}}^{2}\left(\omega_{s}^{2}-\omega_{b}^{2}\right)}{\left(\omega_{s}^{2}-\omega_{b}^{2}\right)^{2}+4 \nu^{2} \omega_{s}^{2}}, \\
& \operatorname{Im}\left[F_{\chi}\right] \approx \frac{2 \omega_{\mathrm{pi}}^{2} \omega_{b} \nu}{\left(\omega_{s}^{2}-\omega_{b}^{2}\right)^{2}+4 \nu^{2} \omega_{s}^{2}},
\end{aligned}
$$

where $\omega_{s}=k_{b} c_{s}$ is the frequency of a plasma ion acoustic wave. This essentially shows that for $\omega_{b}=0, \operatorname{Im}\left[F_{\chi}\right]=0$ and $\phi_{2}=0$ (no energy transfer), but $\operatorname{Re}\left[F_{\chi}\right]=\chi_{e}=$ $1 /\left(k_{b} \lambda_{D}\right)^{2}$ (finite dephasing). This situation can lead to a plasma-based Pockels cell. And on the other hand, when $\omega_{b}=-\omega_{s}$, the moving index modulation resonantly drives an ion acoustic wave and leads to $\operatorname{Re}\left[F_{\chi}\right]=0$ and $\operatorname{Im}\left[F_{\chi}\right]=-\omega_{\mathrm{pi}}^{2} /\left(\omega_{s} \nu\right)$, with $\phi_{2}=-\pi / 2$ : this is the wellknown mechanism of "crossed-beam energy transfer" in plasmas [12-16], which in this case is the physical origin for our plasma-based polarizer concept. Note that when designing experiments in plasmas, one might want to operate in the same regime of fluid and linear interactions that was used in Refs. [3,4], relying on long interaction distances at moderate laser intensities to satisfy $n_{2} I_{0} L / \lambda=$ $O(1)$ while keeping $\delta n / n \ll 1$ (i.e., small density perturbation) in order to avoid nonlinear and kinetic behaviors of the driven plasma waves.

Some practical limitations for these concepts need to be emphasized. First, we neglected the "self-action" processes; i.e., we ignored the terms $\left|E_{0}\right|^{2}$ and $\left|E_{1}\right|^{2}$ from the right-hand side of Eq. (1). For SBS in plasmas or induced by electrostriction, these should not significantly contribute since the force at the origin of the index modulation (electrostrictive or ponderomotive) is proportional to the gradient of the intensity, $\propto \nabla\left\langle\tilde{\boldsymbol{E}}^{2}\right\rangle$. However, if the intensities are too high and/or the pulse duration too short, this could still lead to an overall variation of the index and trigger filamentation of the beams and other nonlinear effects, depending on the phase front qualities of the beams.

The other aspect that will constrain the design of experiments is the fact that our analysis is $2 \mathrm{D}$, in the plane of incidence. The results presented here can easily be generalized to 3D; however, this would require either that the auxiliary has a "square" spot profile, so that the results presented here remain identical for any $z$ within the auxiliary's envelope, or that the probe's spot size is smaller than the auxiliary's, so that variations of the auxiliary intensity along $z$ are negligible across the probe's spot profile. The latter case could go against the main benefit of this geometry, which is to allow manipulation of a probe using a less intense auxiliary beam-unless the probe-toauxiliary intensity ratio is larger than the auxiliary-to-probe spot size ratio, such that the probe power still exceeds the auxiliary's. On the other hand, this type of polarization mixing might offer yet new opportunities if one uses less conventional optical structures for the light waves, such as Bessel beams, orbital angular momentum, etc.

Finally, we propose a few examples for practical realizations of these concepts. First, a polarizer can be designed by using a high-SBS gain medium such as an FC-40 liquid, which has been used in recent SBS amplification experiments $[17,18]$. The measured SBS gain is $\sim 2 \mathrm{~cm} / \mathrm{GW}$ for $1053 \mathrm{~nm}$ light, for $\mathrm{a} \simeq 1.5 \mathrm{GHz}$ frequency shift between the auxiliary and probe. Therefore, in the absence of $2 \mathrm{D}$ 
(or 3D) effects, using an auxiliary beam at $2 \mathrm{GW} / \mathrm{cm}^{2}$ frequency shifted to the Stokes frequency to allow energy transfer from the probe to the auxiliary along the $z$ direction (cf. Fig. 4) over a $1 \mathrm{~cm}$ interaction length should lead to an extinction of $\exp [-4] \simeq 1.8 \%$. For a $90^{\circ}$ crossing geometry, this could, for example, be realized using a $2 \mathrm{GW}$ auxiliary beam with a cross section of $1 \mathrm{~cm}^{2}$.

Next, we give the example of a plasma wave plate in high-energy density environments such as those encountered in experiments at the National Ignition Facility (NIF) $[19,20]$. Assuming typical electron and ion plasma temperatures of 3 and $1 \mathrm{keV}$, an electron density of $10^{21} \mathrm{~cm}^{-3}$ (i.e., $\sim 10 \%$ of critical at $351 \mathrm{~nm}$ ), and a helium plasma, a quarter-wave plate can be achieved for an auxiliary beam intensity of $3 \times 10^{14} \mathrm{~W} / \mathrm{cm}^{2}$ if the interaction length is $1.5 \mathrm{~mm}$. These parameters are very typical of NIF experiments, where the beams are typically in the mid$10^{14} \mathrm{~W} / \mathrm{cm}^{2}$ intensity range and the laser spot sizes are between 0.7 and $1.8 \mathrm{~mm}$. Furthermore, having 192 beams means 18,336 possible pairs, of which we calculated that 280 individual pairs of beams are crossing at $90^{\circ} \pm 1^{\circ}$, meaning that a plasma wave plate with a perpendicular crossing arrangement like in Fig. 4 should be readily accessible within the current experimental conditions at the NIF.

\section{SUMMARY AND CONCLUSION}

In conclusion, we have presented the theory of two-wave mixing in an arbitrary medium with optical nonlinearity. This extends the concepts of polarization control in the linear regime, which we already demonstrated in plasmas [2], to a very wide variety of nonlinear optical media such as gases or fluids.

While the linear theory describes the dynamic manipulation of a probe's polarization using a more intense auxiliary beam, here we investigated the nonlinear regime of polarization mixing, where the probe is intense enough to alter the polarization of the auxiliary. We have derived a fully analytical solution of the polarization mixing for a $90^{\circ}$ crossing geometry, and showed that this geometry, combined with an $s$-polarized auxiliary, allows full polarization control of a probe of arbitrary intensity-even exceeding the auxiliary's intensity. This can lead to a Pockels cell concept, where the amount of birefringence can be controlled dynamically by adjusting the intensity of the auxiliary, or a polarizer, where the extinction ratio is also controlled dynamically via the auxiliary's intensity. Achieving these effects requires the product of the nonlinear index perturbation and the interaction distance (normalized to the laser wavelength) to be of order 1, i.e., $n_{2} I_{0} L_{\text {int }} / \lambda_{0} \sim O(1)$, where the interaction length will typically be related to the auxiliary's spot size and crossing angle, $L_{\text {int }}=w_{0} / \sin (\psi)$ (with $\psi=\pi / 2$ for a nonlinear interaction allowing $I_{1} \geq I_{0}$, or $\psi \ll 1$ for operation in the linear regime). These concepts could lead to ultrafast polarization manipulation schemes if the medium's response time is fast enough (e.g., via ionization in a gas, cf. Refs. [8-10]), or to plasma-based optics with essentially unlimited tolerance to optics damage [11,21-23].

\section{ACKNOWLEDGMENTS}

The authors are grateful to J. E. Heebner, M. R. Edwards, L. Friedland, G. Marcus, and R. Trines for fruitful discussions. This work was performed under the auspices of the U.S. Department of Energy by Lawrence Livermore National Laboratory under Contract No. DE-AC5207NA27344, and was funded by the Laboratory Research and Development Program at LLNL under Project Tracking Code No. 18-ERD-046 and the NSFBSF Grant No. 1803874 (BSF No. 6079). This document was prepared as an account of work sponsored by an agency of the U.S. government. Neither the U.S. government nor Lawrence Livermore National Security, LLC, nor any of their employees makes any warranty, expressed or implied, or assumes any legal liability or responsibility for the accuracy, completeness, or usefulness of any information, apparatus, product, or process disclosed, or represents that its use would not infringe privately owned rights. The views and opinions of authors expressed herein do not necessarily state or reflect those of the U.S. government or Lawrence Livermore National Security, LLC, and shall not be used for advertising or product endorsement purposes.

[1] R. W. Boyd, Nonlinear Optics, 3rd ed. (Academic Press, Inc., Orlando, FL, 2008).

[2] P. Michel, L. Divol, D. Turnbull, and J. D. Moody, Dynamic Control of the Polarization of Intense Laser Beams via Optical Wave Mixing in Plasmas, Phys. Rev. Lett. 113, 205001 (2014).

[3] D. Turnbull, P. Michel, T. Chapman, E. Tubman, B. B. Pollock, C. Y. Chen, C. Goyon, J. S. Ross, L. Divol, N. Woolsey, and J. D. Moody, High Power Dynamic Polarization Control Using Plasma Photonics, Phys. Rev. Lett. 116, 205001 (2016).

[4] D. Turnbull, C. Goyon, G. E. Kemp, B. B. Pollock, D. Mariscal, L. Divol, J. S. Ross, S. Patankar, J. D. Moody, and P. Michel, Refractive Index Seen by a Probe Beam Interacting with a Laser-Plasma System, Phys. Rev. Lett. 118, 015001 (2017).

[5] W. L. Kruer, The Physics of Laser Plasma Interactions (Westview Press, Boulder, CO, USA, 2003).

[6] T. Speziale, Stimulated Brillouin Sidescattering with Strong Ion Wave Damping, Phys. Fluids 27, 2583 (1984).

[7] C. J. McKinstrie, J. S. Liu, R. E. Giacone, and H. X. Vu, Three-Dimensional Analysis of the Power Transfer between Crossed Laser Beams, Phys. Plasmas 7, 3 (1996).

[8] J. K. Wahlstrand, J. H. Odhner, E. T. McCole, Y.-H. Cheng, J. P. Palastro, R. J. Levis, and H. M. Milchberg, Effect of Two-Beam Coupling in Strong-Field Optical Pump-Probe Experiments, Phys. Rev. A 87, 053801 (2013). 
[9] J. K. Wahlstrand and H. M. Milchberg, Effect of a Plasma Grating on Pump-Probe Experiments Near the Ionization Threshold in Gases, Opt. Lett. 36, 3822 (2011).

[10] L. Shi, W. Li, Y. Wang, X. Lu, L. Ding, and H. Zeng, Generation of High-Density Electrons Based on Plasma Grating Induced Bragg Diffraction in Air, Phys. Rev. Lett. 107, 095004 (2011).

[11] H. Milchberg, Indestructible Plasma Optics, Phys. Today 72, No. 6, 70-71 (2019).

[12] W. L. Kruer, S. C. Wilks, B. B. Afeyan, and R. K. Kirkwood, Energy Transfer between Crossing Laser Beams, Phys. Plasmas 3, 382 (1996).

[13] P. Michel, L. Divol, E. A. Williams, S. Weber, C. A. Thomas, D. A. Callahan, S. W. Haan, J. D. Salmonson, S. Dixit, D. E. Hinkel, M. J. Edwards, B. J. MacGowan, J. D. Lindl, S. H. Glenzer, and L. J. Suter, Tuning the Implosion Symmetry of ICF Targets via Controlled Crossed-Beam Energy Transfer, Phys. Rev. Lett. 102, 025004 (2009).

[14] I. V. Igumenshchev et al., Crossed-Beam Energy Transfer in Direct-Drive Implosions, Phys. Plasmas 19, 056314 (2012).

[15] S. H. Glenzer et al., Symmetric Inertial Confinement Fusion Implosions at Ultra-High Laser Energies, Science 327, 1228 (2010).

[16] J. D. Moody et al., Multistep Redirection by Cross-Beam Power Transfer of Ultrahigh-Power Lasers in a Plasma, Nat. Phys. 8, 344 (2012).

[17] H. Yoshida, T. Hatae, H. Fujita, M. Nakatsuka, and S. Kitamura, A High-Energy 160-ps Pulse Generation by
Stimulated Brillouin Scattering from Heavy Fluorocarbon Liquid at $1064 \mathrm{~nm}$ Wavelength, Opt. Express 17, 13654 (2009).

[18] H. Yuan, Y. Wang, Z. Lu, and Z. Zheng, Active Frequency Matching in Stimulated Brillouin Amplification for Production of a 2.4 J, 200 ps Laser Pulse, Opt. Lett. 43, 511 (2018).

[19] M. L. Spaeth et al., Description of the NIF Laser, Fusion Sci. Technol. 69, 25 (2016).

[20] E. I. Moses, J. D. Lindl, M. L. Spaeth, R. W. Patterson, R. H. Sawicki, L. J. Atherton, P. A. Baisden, L. J. Lagin, D. W. Larson, B. J. MacGowan, G. H. Miller, D. C. Rardin, V. S. Roberts, B. M. Van Wonterghem, and P. J. Wegner, Overview: Development of the National Ignition Facility and the Transition to a User Facility for the Ignition Campaign and High Energy Density Scientific Research, Fusion Sci. Technol. 69, 1 (2016).

[21] D. J. Stark, C. Bhattacharjee, A. V. Arefiev, T. Toncian, R. D. Hazeltine, and S. M. Mahajan, Relativistic Plasma Polarizer: Impact of Temperature Anisotropy on Relativistic Transparency, Phys. Rev. Lett. 115, 025002 (2015).

[22] G. Lehmann and K. H. Spatschek, Plasma-Based Polarizer and Waveplate at Large Laser Intensity, Phys. Rev. E 97, 063201 (2018).

[23] K. Qu, Q. Jia, and N. J. Fisch, Plasma q-Plate for Generation and Manipulation of Intense Optical Vortices, Phys. Rev. E 96, 053207 (2017). 